\title{
Parabola - parabola combined method
}

\author{
Z. Szabó
}




\title{
PARABOLA-PARABOLA COMBINED METHOD
}

\author{
Z. SZABÓ
}

\begin{abstract}
AвSTRACT. By using the always convergent method of tangential parabolas (but not using any interval-arithmetic tool), a combined root-finding iterative algorithm is given which provides a quadratically convergent descending sequence of compact real intervals $J_{n}$ containing a simple zero of a twice differentiable real function defined on $J_{0}$.
\end{abstract}

Mathematics Subject Classification: 65H05

Keywords: Nonlinear equation, iteration, always convergent method, tangential parabola, decreasing sequence of compact intervals

\section{Preliminaries, convergence, error estimation}

$\mathbf{T}_{\mathrm{N}}^{\mathrm{N}}$ [1] AND [2], we described two combined root-finding algorithms based on Newton's method and the method of tangential parabolas which is always convergent. The information used by the method in [1] comprises 4 Horner units: $f\left(a_{n}\right), f^{\prime}\left(a_{n}\right)$, $f\left(b_{n}\right)$, and $f^{\prime}\left(b_{n}\right)$. This number is reduced to 2 in [2]. Keeping this number 2 of Horner's units used, we aim here at finding a faster combined method generating an interval sequence with the known features. The conditions (2), (3), and (6) in [2] will be replaced by the assumption

$$
m_{2} \leq\left|f^{\prime \prime}(x)\right| \text { in } I=[a, b] \subset \mathbb{R} \text {, for some } m_{2}>0,
$$

provided $f^{\prime \prime}$ does not change its sign in $I$.

In this new algorithm, the tangential straight line in the "Newton-Parabola" combined method described in [2] will be replaced by another tangential parabola lying "outside" the graph of $f$ (the area between the curve of $f$ and the $x$-axis is considered to be "inside"). For this "outer parabola," we first prove our

Lemma 1. Assume that the function $f: I=[a, b] \rightarrow \mathbb{R}$ is twice differentiable in the compact interval $I \subset \mathbb{R}$, assumption (1.1) holds, and $f\left(x_{0}\right) \neq 0$ for some $x_{0}$ in $I$. Then, for the tangential parabola

$$
p(x)=f\left(x_{0}\right)+f^{\prime}\left(x_{0}\right)\left(x-x_{0}\right)-\frac{1}{2} m_{2} \operatorname{sign}\left(f\left(x_{0}\right)\right)\left(x-x_{0}\right)^{2},
$$

Supported by the Hungarian Ministry of Culture and Education under Grant No. FKFP0429. 
the difference $p(x)-f(x)$ is non-negative (resp., non-positive), provided $f\left(x_{0}\right)$ is positive (resp., negative), and $f$ is concave from below (resp., above) in $I$.

In other words, we claim that $f\left(x_{0}\right)(p(x)-f(x)) \geq 0$ in $I$, provided the assumptions are fulfilled.

Proof of Lemma 1. Let $f\left(x_{0}\right)>0$. Then we have

$$
\begin{gathered}
p(x)=f\left(x_{0}\right)+f^{\prime}\left(x_{0}\right)\left(x-x_{0}\right)-\frac{1}{2} m_{2}\left(x-x_{0}\right)^{2}, \\
f(x)=f\left(x_{0}\right)+f^{\prime}\left(x_{0}\right)\left(x-x_{0}\right)-\frac{1}{2} f^{\prime \prime}(c)\left(x-x_{0}\right)^{2}
\end{gathered}
$$

for some $c \in\left(x_{0}, x\right)$, and $0<m_{2} \leq-f^{\prime \prime}(x), x \in I$. Thus,

$$
p(x)-f(x)=\frac{1}{2}\left(-f^{\prime \prime}(c)-m_{2}\right)\left(x-x_{0}\right)^{2} \geq 0, \quad x \in I,
$$

because $-f^{\prime \prime}(c)=\left|f^{\prime \prime}(c)\right| \geq m_{2}$. When $f\left(x_{0}\right)<0$, we have $m_{2} \leq f^{\prime \prime}(x), x \in I$, and the proof is similar, namely, in $p(x)-f(x)$, the term $-f^{\prime \prime}(c)$ will be replaced by $-m_{2}$.

The convexity assumptions in Lemma 1 can be summarized as follows:

$$
0<m_{2} \leq-\operatorname{sign}\left(f\left(x_{0}\right)\right) f^{\prime \prime}(x), x \in I .
$$

We need now a more accurate notation for the iteration function of the "tangential parabola" method (briefly, TP-method) described in [1, pp. 581-582]. Let

$$
F_{T P}(x, r, M):= \begin{cases}x+s f^{\prime}(x) / M+r\left(2|f(x)| / M+\left(f^{\prime}(x) / M\right)^{2}\right)^{1 / 2} \\ x \text { if } s=0 . & \text { if } s=f\left(x_{0}\right) \neq 0 ;\end{cases}
$$

In order to define our combined "parabola-parabola" method (PP-method for short), we assume that the following condition is satisfied:"

(A) The nonlinear function $f: I=[a, b] \rightarrow \mathbb{R}$ is twice differentiable on $I^{\dagger}$, the inequalities $\left|f^{\prime \prime}(x)\right| \leq M_{2} \neq 0, x \in I$, and $f(a) f(b)<0$ are fulfilled, and $f^{\prime \prime}$ does not change its sign on $I$ *

In addition, we assume that (1.1) holds as well. Let, e. g. $f(a)>0$ and $f^{\prime \prime}(x)<0$. Thus, $f(a) f^{\prime \prime}(x)<0$. (It does not matter whether $f^{\prime}(a)$ is negative or non-negative.) Starting with the points $a_{0}=a$ and $b_{0}=b$, we construct the sequences

$$
a_{n+1}=F_{T P}\left(a_{n}, 1, M_{2}\right), \quad b_{n+1}=\min \left\{F_{T P}\left(a_{n}, 1, m_{2}\right), b\right\},
$$

*Actually, assumption (A) is the same as (1) in [2].

${ }^{\dagger}$ At the endpoints of $I$, the one-sided derivatives are to be considered.

${ }^{\ddagger}$ It follows from (A) that $f^{\prime}$ is monotonic on $I, f$ has a unique zero (say, $\alpha$ ) in $I$, and $\alpha$ is a simple zero: $f(\alpha)=0, f^{\prime}(\alpha) \neq 0$. 
for $n=0,1,2, \ldots$. Since $\alpha$ is the only zero of $f$ in $(a, b)$ and, according to [1, pp. 581582], $F_{T P}$ is an always convergent iteration function: $F_{T P} \in A(f, I)$, and we have $\lim _{n \rightarrow \infty} a_{n}=\alpha$. So, after finitely many $($ say, $N$ ) steps,

$$
b_{N}=\min \left\{F_{T P}\left(a_{N-1}, 1, m_{2}\right), b\right\}=F_{T P}\left(a_{N-1}, 1, m_{2}\right) \leq b
$$

and, for $n \geq N$, the formulae (1.2) take the form

$$
a_{n+1}=F_{T P}\left(a_{n}, 1, M_{2}\right), \quad b_{n+1}=F_{T P}\left(a_{n}, 1, m_{2}\right), \quad n=0,1,2, \ldots
$$

In this manner, we obtain the sequence of compact real intervals

$$
J_{n}=\left[a_{n}, b_{n}\right] ; \quad n=0,1,2, \ldots ; \quad\left(J_{0}=I\right) .
$$

It follows that

$$
J_{n+1} \subset J_{n}, n=0,1,2, \ldots, \quad \text { and } \bigcap_{n=0}^{\infty} J_{n}=\alpha \in I, \quad f(\alpha)=0, \quad f^{\prime}(\alpha) \neq 0 .
$$

In general, we start our iteration from the endpoint $E \in\{a, b\}$ for which the inequality

$$
f(E) f^{\prime \prime}(x)<0
$$

is fulfilled. If, by using formulae similar to (1.3), $a_{n}$ (or $b_{n}$ ) does not belong to $I$, then we choose the nearest endpoint of $I$ to be $a_{n}$ (or $b_{n}$ ). This nearest endpoint will be $\hat{E}=\{a, b\} \backslash\{E\}$. In particular, if $f(a) f^{\prime \prime}(x)<0$, then $E=a, \hat{E}=b, r=1$, and (1.2) remains the same; after a finite number of steps, we reach a point of iteration $a_{n}$ such that $f^{\prime}$ keeps its sign in $\left[a_{n}, b\right]$; if $f(b) f^{\prime \prime}(x)<0$, then $E=b, \hat{E}=a, r=-1$, and (1.2) becomes

$$
a_{n+1}=\max \left\{F_{T P}\left(b_{n},-1, m_{2}\right), a\right\}, \quad b_{n+1}=F_{T P}\left(b_{n},-1, M_{2}\right),
$$

for $n=0,1,2, \ldots$ In this case, after finitely many steps, we reach a point $b_{n}$ such that $f^{\prime}$ keeps its sign in $\left[a, b_{n}\right]$. On the other hand, we have $r=\operatorname{sign}((a+b) / 2-E)=$ $\operatorname{sign}(a+b-2 E)$. An error estimate for our PP-method is contained in the following

Lemma 2. Assume that conditions (1.1) and (A) are fulfilled, and $f^{\prime}$ keeps its sign in $I=J_{0}$. Then, for the diameters of the intervals $J_{n}$, we have the estimate

$$
d\left(J_{n+1}\right) \leq C\left(d\left(J_{n}\right)\right)^{2}, \quad n=0,1,2, \ldots,
$$

where

$$
\begin{gathered}
C=\frac{1}{2} u^{2}\left(M_{2} v^{-3}-m_{2}\left(u^{2}+2|f(E)| m_{2}\right)^{-3 / 2}\right), \\
u=\left|f^{\prime}(\hat{E})\right|=\max \left\{\left|f^{\prime}(a)\right|,\left|f^{\prime}(b)\right|\right\}, \\
v=\left|f^{\prime}(E)\right|=\min \left\{\left|f^{\prime}(a)\right|,\left|f^{\prime}(b)\right|\right\}
\end{gathered}
$$

and $E, \hat{E}$ are defined by the relations $\{E, \hat{E}\}=\{a, b\}$ and (1.4). 
Proof. Let, e. g., $f(a)>0, f^{\prime \prime}(x)<0$. Then $E=a, \hat{E}=b$. By using the formulas

$$
a_{n+1}=a_{n}+f^{\prime}\left(a_{n}\right) / M_{2}+\left(2 f\left(a_{n}\right) / M_{2}+\left(f^{\prime}\left(a_{n}\right) / M_{2}\right)^{2}\right)^{1 / 2}
$$

and

$$
b_{n+1}=a_{n}+f^{\prime}\left(a_{n}\right) / m_{2}+\left(2 f\left(a_{n}\right) / m_{2}+\left(f^{\prime}\left(a_{n}\right) / m_{2}\right)^{2}\right)^{1 / 2},
$$

we get

$$
\begin{aligned}
d\left(J_{n+1}\right)= & b_{n+1}-a_{n+1}=f^{\prime}\left(a_{n}\right)\left(1 / m_{2}-1 / M_{2}\right) \\
& +\left(2 f\left(a_{n}\right) / m_{2}+\left(f^{\prime}\left(a_{n}\right) / m_{2}\right)^{2}\right)^{1 / 2} \\
& -\left(2 f\left(a_{n}\right) / M_{2}+\left(f^{\prime}\left(a_{n}\right) / M_{2}\right)^{2}\right)^{1 / 2} \\
= & 1 / m_{2}\left(y+F\left(m_{2}\right)\right)-1 / M_{2}\left(y+F\left(M_{2}\right)\right)
\end{aligned}
$$

where $y=f^{\prime}\left(a_{n}\right)=-|y|<0, q=2 f\left(a_{n}\right)$ and $F(m)=\left(y^{2}+q m\right)^{1 / 2}$. We take $F^{\prime}(m)=\frac{1}{2} q\left(y^{2}+q m\right)^{-1 / 2}$ and $F^{\prime \prime}(m)=-\frac{1}{4} q^{2}\left(y^{2}+q m\right)^{-3 / 2}$. By using Taylor's formula, we obtain

$$
\begin{aligned}
F(m) & =F(0)+F^{\prime}(0) m+\frac{1}{2} F^{\prime \prime}(w) m^{2} \\
& =|y|+q m /(2|y|)-\frac{1}{8} q^{2} m^{2}\left(y^{2}+q w\right)^{-3 / 2},
\end{aligned}
$$

where $w \in(0, m)$. Then (1.6) takes the form

$$
\begin{aligned}
d\left(J_{n+1}\right)= & \frac{1}{m_{2}}\left(y+|y|+q \frac{m_{2}}{2|y|}-\frac{1}{8} q^{2} m_{2}^{2}\left(y^{2}+q w\right)^{-3 / 2}\right) \\
& -\frac{1}{M_{2}}\left(y+|y|+q \frac{M_{2}}{2|y|}-\frac{1}{8} q^{2} M_{2}^{2}\left(y^{2}+q W\right)^{-3 / 2}\right) \\
= & \frac{q^{2}}{8}\left(M_{2}\left(y^{2}+q W\right)^{-3 / 2}-m_{2}\left(y^{2}+q w\right)^{-3 / 2}\right) \\
= & \frac{1}{2} f^{2}\left(a_{n}\right)\left(M_{2}\left(f^{\prime 2}\left(a_{n}\right)+2 f\left(a_{n}\right) W\right)^{-3 / 2}-m_{2}\left(f^{\prime 2}\left(a_{n}\right)+2 f\left(a_{n}\right) w\right)^{-3 / 2}\right),
\end{aligned}
$$

where $w \in\left(0, m_{2}\right)$ and $W \in\left(0, M_{2}\right)$. The factor $f^{2}\left(a_{n}\right)$ on the right-hand side can be replaced by $f^{\prime 2}\left(t_{n}\right)\left(\alpha-a_{n}\right)^{2}$ for some $t_{n} \in\left(a_{n}, \alpha\right)$ because, by the mean value theorem, $f(\alpha)-f\left(a_{n}\right)=f^{\prime}\left(t_{n}\right)\left(\alpha-a_{n}\right)$. Thus, one can estimate $d\left(J_{n+1}\right)$ as follows:

$$
\begin{aligned}
d\left(J_{n+1}\right) & \leq \frac{1}{2} f^{\prime 2}\left(t_{n}\right)\left(\alpha-a_{n}\right)^{2}\left(M_{2} /\left|f^{\prime}\left(a_{n}\right)\right|^{3}-m_{2}\left(f^{\prime 2}\left(a_{n}\right)+2 f\left(a_{n}\right) m_{2}\right)^{-3 / 2}\right) \\
& \leq \frac{1}{2} f^{\prime 2}(b)\left(\alpha-a_{n}\right)^{2}\left(M_{2} /\left|f^{\prime}(a)\right|^{3}-m_{2}\left(f^{\prime 2}(b)+2 f(a) m_{2}\right)^{-3 / 2}\right) \\
& =\frac{1}{2} u^{2}\left(\alpha-a_{n}\right)^{2}\left(M_{2} / v^{3}-m_{2}\left(u^{2}+2|f(E)| m_{2}\right)^{-3 / 2}\right) \\
& \leq C\left(b_{n}-a_{n}\right)^{2}=C\left(d\left(J_{n}\right)\right)^{2} .
\end{aligned}
$$


In the remaining three cases corresponding to the signs of $f(a)$ and $f^{\prime \prime}(x)$, the proofs are quite similar.

Remark 1. Assumptions (1.1) and (A) imply that

$$
v<u<\left(u^{2}+2|f(E)| m_{2}\right)^{1 / 2},
$$

so $C>0$.

Remark 2. A geometrical representation shows that our PP-method is faster than the combined method described in [2]. (Namely, the "outer parabola" lies below (resp., above) the tangential line of Newton's method if $f\left(a_{n}\right)>0$ (resp., $f\left(a_{n}\right)<0$ ). This fact is also reflected in the error estimates because

$$
C=K-\frac{1}{2} u^{2} m_{2}\left(u^{2}+2|f(E)| m_{2}\right)^{-3 / 2} .
$$

Remark 3. If $\left|f\left(a_{n}\right)\right| \ll 1$ (i. e., $a_{n}$ is close to $\alpha$ ), then

$$
C \approx \frac{1}{2} u^{2}\left(M_{2} / v^{3}-m_{2} / u^{3}\right)=K-\frac{1}{2} m_{2} / u
$$

where $K$ is the error constant in [2].

Remark 4. For the endpoint $\hat{E}$, we have $f(\hat{E}) f^{\prime \prime}(x)>0$. According to the Fourier conditions, the Newton method can be used, starting at $\hat{E}$ (like in [1]).

The results above can be summarized in our main

Theorem 1. If assumptions (1.1) and (A) hold, then the sequence of compact real intervals $J_{n}=\left[a_{n}, b_{n}\right], n=0,1,2, \ldots$, generated by the combined PP-method described above has the following properties:

$1^{\circ} J_{n+1} \subset J_{n}, n=0,1,2, \ldots$;

$2^{\circ} \cap_{n=0}^{\infty} J_{n}=\alpha \in I, f(\alpha)=0, f^{\prime}(\alpha) \neq 0$;

$3^{\circ}$ if $f^{\prime}$ keeps its sign on $I=J_{0}$, then, for the diameters of the resulting intervals, we have the estimate

$$
d\left(J_{n+1}\right) \leq C\left(d\left(J_{n}\right)\right)^{2}, \quad n=0,1,2, \ldots,
$$

where

$$
\begin{gathered}
C=\frac{1}{2} u^{2}\left(M_{2} v^{-3}-m_{2}\left(u^{2}+2|f(E)| m_{2}\right)^{-3 / 2}\right), \\
u=\left|f^{\prime}(\hat{E})\right|=\max \left\{\left|f^{\prime}(a)\right|,\left|f^{\prime}(b)\right|\right\}, \quad v=\left|f^{\prime}(E)\right|=\min \left\{\left|f^{\prime}(a)\right|,\left|f^{\prime}(b)\right|\right\}, \\
\text { and } E, \hat{E} \text { are defined by the relations }\{E, \hat{E}\}=\{a, b\} \text { and }(1.4) .
\end{gathered}
$$




\section{Algorithm AND SOMe NUMERICAL EXAMPLes}

The algorithm for the PP-method can be given by the following Boolean function procedure $P P M$. The input parameters are $a, b$, eps, $m 2, M 2, f$ and $f 1$ (corresponding to $f^{\prime}$ ). If they fulfil the requirements $a<b$, eps $>0,0<m 2 \leq M 2$ and $f(a) f(b) \leq 0$, then $P P M$, the identifier of our Boolean procedure, will have the logical value true, otherwise $P P M$ will be false. The other output parameters are the endpoints of the resulting interval $[A, B]$ including $\alpha$ (the zero of $f$ ) and having a diameter less than eps, provided $P P M=$ true. Otherwise, $[A, B]$ will be the initial interval $[a, b]$.

The subroutines/procedures for the functions $f$ and $f^{\prime}$ have to be defined by the user.

$S U B$ is a real function procedure with the formal input parameter $L$. The actual input parameters are $m_{2}$ and $M_{2}$. This procedure actually calculates the value of $F_{T P}(z, r, L)$, the tangential parabola iterate of $z$ for $L \in\left\{m_{2}, M_{2}\right\}$, and puts it into the output parameter $S U B$.

The integer variable $k$ contains the information on convexity of the graph of $f$. If $k=1$, then $(f(a)+f(b)) / 2>f((a+b) / 2)$ and $f$ is convex; if $k=-1$, then $f$ is concave. Thus, $k=\operatorname{sign}(f(a)+f(b)-2 f((a+b) / 2))$. (We have $k \neq 0$, i. e., $f$ is non-linear because $m_{2}>0$.)

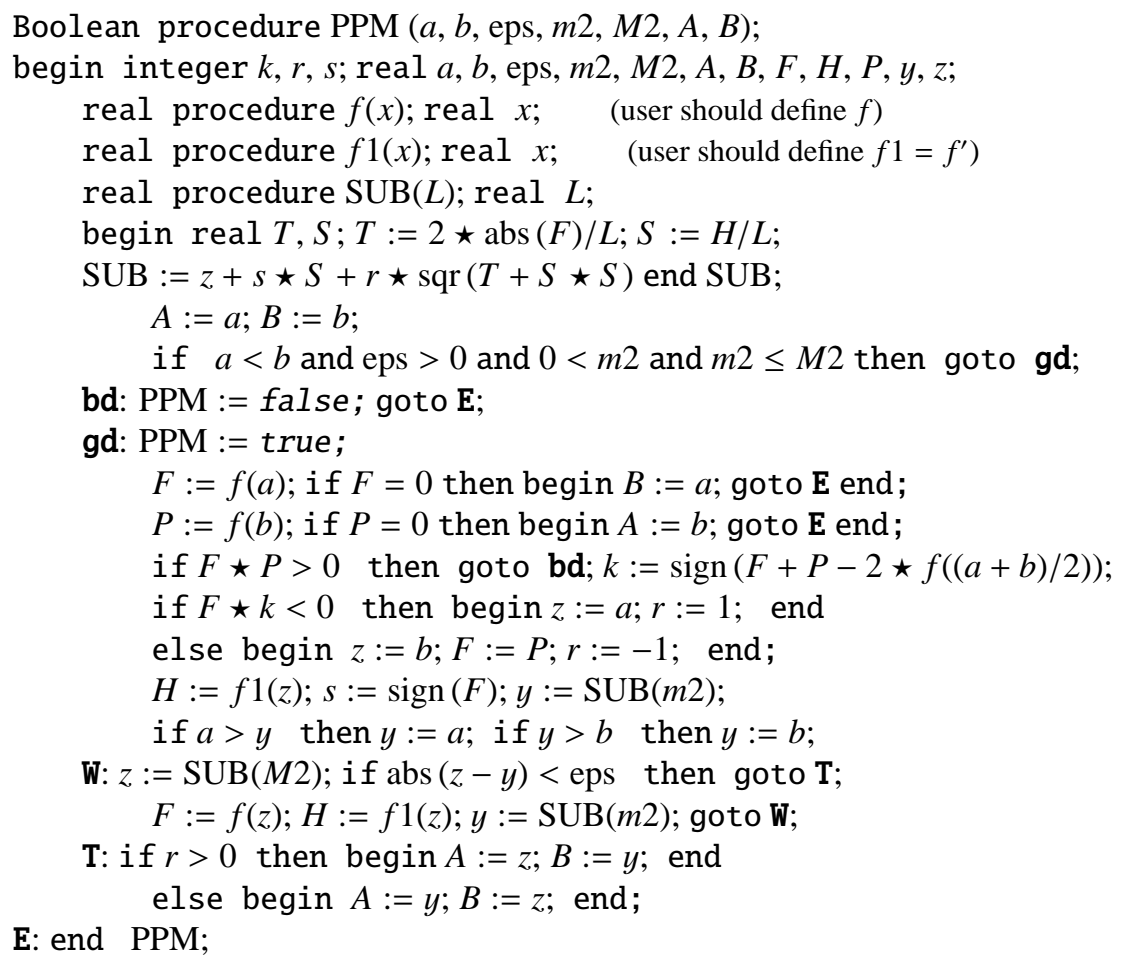


Some numerical examples can be found in the following tables, where the correct digits are underlined.

Example 1. $f(x)=x-e^{-x}, a=0, b=1(=E), M_{2}=1, m_{2}=0.35$;

\begin{tabular}{|l|l|l|l|}
\hline$n$ & $a_{n}$ & $b_{n}$ & $b_{n}-a_{n}$ \\
\hline \hline 0 & 0 & 1 & 1 \\
1 & $\underline{0.56238349331149966899}$ & $\underline{0.59719164168881961091}$ & $3 \cdot 10^{-2}$ \\
2 & $\underline{0.56708373561334769584}$ & $\underline{0.56727015271662188072}$ & $2 \cdot 10^{-4}$ \\
3 & $\underline{0.56714328929501556755}$ & $\underline{0.56714329263260011212}$ & $3 \cdot 10^{-9}$ \\
4 & $\underline{0.56714329040978387265}$ & $\underline{0.56714329040978387368}$ & $1 \cdot 10^{-18}$ \\
5 & $\underline{0.56714329040978387300}$ & $\underline{0.56714329040978387300}$ & $1 \cdot 10^{-37}$ \\
\hline
\end{tabular}

Example 2. $f(x)=\tan ^{-1} x-2.6+\sqrt{x}, a=1, b=4(=E), M_{2}=0.75, m_{2}=0.035$;

\begin{tabular}{|l|l|l|l|}
\hline$n$ & $a_{n}$ & $b_{n}$ & $b_{n}-a_{n}$ \\
\hline \hline 0 & 1 & 4 & 3 \\
1 & 1.89970378394449937319 & $\underline{2.96088085705371547709}$ & 1 \\
2 & $\underline{2.06567277560842922080}$ & $\underline{2} .36679176536415266599$ & $3 \cdot 10^{-1}$ \\
3 & $\underline{2.13894682376643847337}$ & $\underline{2.16810797543185165243}$ & $3 \cdot 10^{-2}$ \\
4 & $\underline{2.14658693492719685263}$ & $\underline{2.14689875120987042863}$ & $3 \cdot 10^{-4}$ \\
5 & $\underline{2.14666632870554397592}$ & $\underline{2.14666636586609630990}$ & $4 \cdot 10^{-8}$ \\
6 & $\underline{2.14666633811284909659}$ & $\underline{2.14666633811284962657}$ & $5 \cdot 10^{-16}$ \\
7 & $\underline{2.14666633811284923074}$ & $\underline{2.14666633811284923074}$ & $1 \cdot 10^{-31}$ \\
\hline
\end{tabular}

Example 3. $f(x)=1-x-\sin x, a=0.01, b=1(=E), M_{2}=0.842, m_{2}=0.0099$;

\begin{tabular}{|l|l|l|l|}
\hline$n$ & $a_{n}$ & $b_{n}$ & $b_{n}-a_{n}$ \\
\hline \hline 0 & 0.01 & 1 & 1 \\
1 & $\underline{0.45465326096563166766}$ & $\underline{0.51736453936087952833}$ & $6 \cdot 10^{-2}$ \\
2 & $\underline{0.51096815380042764464}$ & $\underline{0.51097723467313242901}$ & $9 \cdot 10^{-6}$ \\
3 & $\underline{0.51097342938671630865}$ & $\underline{0.51097342938993405418}$ & $3 \cdot 10^{-12}$ \\
4 & $\underline{0.51097342938856910952}$ & $\underline{0.51097342938856910952}$ & $4 \cdot 10^{-25}$ \\
\hline
\end{tabular}

\section{REFERENCES}

[1] Szabó, Z.: Combined iteration method for solving equations, Colloquia Math. Soc. J. Bolyai, 50, Numerical Methods, Miskolc, 1986, pp. 581-587.

[2] Szaвó, Z.: Newton-parabola combined method for solving equations, In: Computational \& Applied Mathematics, I. Algorithms and Theory, North-Holland, Amsterdam, 1992, pp. 447-452.

\section{Author's Address}

\section{Z. Szabó:}

Sultan Qaboos University, Department of Mathematics and Statistics, P. O. Box 36., Al-Khodh

P. C. 123 , Sultanate of OMan

E-mail address: szabo@squ.edu.om 